\title{
Biodegradation of oxytetracycline by Pleurotus ostreatus mycelium: a mycoremediation technique
}

\author{
Luciana Migliore $^{\mathrm{a}, *}$, Maurizio Fiori ${ }^{\mathrm{b}}$, Anna Spadoni ${ }^{\mathrm{a}, \mathrm{c}}$, Emanuela Galli $^{\mathrm{c}}$ \\ a Dept. Biology, Tor Vergata University, Via della Ricerca Scientifica, 00133 Rome, Italy \\ ${ }^{\mathrm{b}}$ Dept. Veterinary Public Health and Food Safety, Istituto Superiore di Sanità, Viale Regina Elena 299, 00161 Rome, Italy \\ c Institute of Agro-Environment and Forest Biology, National Research Council, Research Area Rome 1, Via Salaria km 29.300, 00015 Monterotondo Rome, Italy
}

\section{A R T I C L E I N F O}

\section{Article history:}

Received 6 December 2011

Received in revised form 18 February 2012

Accepted 21 February 2012

Available online 1 March 2012

\section{Keywords:}

Mycoremediation

Laccase activity

HPLC-UV/DAD analysis

LC-ESI-MS analysis

Oxytetracycline

\begin{abstract}
A B S T R A C T
Oxytetracycline (OTC) is administered in high doses to livestocks and enters the environmental compartments as a consequence of animal waste disposal. As a first step in setting up a useful mycoremediation technique, an OTC lab degradation test was performed in liquid medium using the ligninolytic fungus Pleurotus ostreatus. OTC disappearance in culture medium was clearly evident as early as the third day of exposure onwards, with an almost complete removal after $14 \mathrm{~d}$. The drug removal was mediated by fungal absorption in the mycelia, where the OTC molecule underwent a degradation step, as demonstrated by mass spectrometry analyses. A putative degradation product, ADOTC (2-acetyl-2-decarboxamidooxytetracycline) is proposed. Experimental conditions excluded OTC abiotic degradation; the degradation by extracellular laccase was also experimentally discarded.
\end{abstract}

(C) 2012 Elsevier B.V. All rights reserved.

\section{Introduction}

Oxytetracycline (OTC) is an antimicrobial drug widely used in intensive farming to treat enteric and respiratory diseases [1,2]. Due to oral administration and its pharmacokinetic, OTC dosage is in the range of $\mathrm{g} \mathrm{kg}^{-1}$ medicated feeds and it is found in animal waste at concentrations of $\mathrm{mg} \mathrm{kg}^{-1}$ [3]. As a consequence of waste disposal, the drug enters into the environmental compartments (water, sediments, soil) and can contaminate trophic nets [4-6], determining carry over in food and negative effects on natural systems $[7,8]$, including effects on the microbial community structure and selection of antibiotic-resistant strains [9-11].

To avoid the negative effects, drug degradation and/or removal are a possible solution that can be obtained by bioremediation techniques. Fungi have not extensively investigated for biotransformation of antibiotics [12]. Some of them have been applied in polycyclic aromatic compounds bioremediation and dyes decolorization [13-15]. Wen et al. [16,17] studied the degradation of tetracycline and OTC by crude ligninolytic enzymes (lignin peroxidase and manganese peroxidase) prepared from Phanerochaete chrysosporium, with positive results. Their studies were conducted in vitro and did not include tests on another important ligninolytic enzyme, the laccase, aplenty produced by white-rot fungi.

\footnotetext{
* Corresponding author. Tel.: +39 067259 5984; fax: +39 062023500.

E-mail address: luciana.migliore@uniroma2.it (L. Migliore).
}

In a previous study, Galli et al. [18] utilized P. ostreatus SMR684 in mycoremediation experiments for the degradation of creosote components. This strain is known to produce laccase both in the presence of lignocellulosic substrates and constitutively, in some phases of its life cycle [19].The aim of this study is to investigate the ability of the fungus $P$. ostreatus SMR684 to degrade OTC. To this end in a laboratory model, living mycelia were challenged with different OTC concentrations. A positive result could represent the first step of a mycoremediation technique on a pilot scale.

\section{Materials and methods}

\subsection{Organism and culture conditions}

P. ostreatus (Jacq.) P. Kumm. mycelium, strain SMR684, is stored in minimal growth at $+5^{\circ} \mathrm{C}$ in the Edible Saprophytic Mushrooms collection of IBAF-CNR, Rome, Italy. A small fraction of mycelium was grown for $2 \mathrm{~d}$ in a $250 \mathrm{~mL}$ Erlenmeyer flask containing $100 \mathrm{~mL}$ of a sterilized $3 \%$ malt extract liquid culture medium (Liofilchem s.r.l., Roseto, TE, Italy) on a rotary shaker (100 rpm), under controlled laboratory conditions at $T=25 \pm 1^{\circ} \mathrm{C}$ and Relative Humidity $(\mathrm{RH})=70 \pm 5 \%$.

Pre-inoculum culture was started by aseptically homogenizing the mycelium in an omni-mixer for $3 \mathrm{~s}$ and transferring $50 \mathrm{~mL}$ of mycelial suspension into a flask containing $500 \mathrm{~mL}$ of $3 \%$ malt extract. The mycelium grew in the controlled laboratory conditions for $3 \mathrm{~d}$ then aliquots of this culture were used as inocula. 


\subsection{The experimental plan}

The experimental plan was organized on 6 batches: (1) culture medium ( $3 \%$ malt extract); (2) culture medium $+50 \mu \mathrm{g} \mathrm{mL} \mathrm{m}^{-1} \mathrm{OTC}$; (3) culture medium $+100 \mu \mathrm{g} \mathrm{mL}^{-1}$ OTC; (4) culture medium $+P$. ostreatus; (5) culture medium $+P$. ostreatus $+50 \mu \mathrm{g} \mathrm{mL}^{-1}$ OTC; (6) culture medium $+P$. ostreatus $+100 \mu \mathrm{g} \mathrm{mL}^{-1}$ OTC. Batches from \#1 to 4 are controls, \#5 and \#6 are test batches. Each batch was constituted by 18 Erlenmeyer flasks ( 3 replicates for each day of sampling). The entire experiment has been repeated three times.

A sterilized solution ( $0.2 \mu \mathrm{m}$ filter) of OTC (Sigma, Milan) in deionised water was added to the test cultures up to the final concentrations in the culture media of 50 and $100 \mu \mathrm{g} \mathrm{mL}^{-1}$. Aliquots of $6 \mathrm{~mL}$ of the pre-inoculum culture (corresponding to $14.4 \mathrm{mg}$ of mycelium, dry weight, d.w.) were used as inocula.

All flasks were incubated at $25 \pm 1{ }^{\circ} \mathrm{C}$ and $70 \pm 5 \%$ RH. Samples of each batch were collected at the beginning of the trial (T0) and after 2 (T2), 3 (T3), 7 (T7), 10 (T10) and 14 d (T14). To avoid OTC photodegradation, flasks were entirely covered by aluminium sheets and all experimental procedures were performed under dark. Different samples were obtained: from \#1 to 3 batches only culture media; from \#4 to 6 both mycelia and culture media. Mycelia were separated from culture medium by gauze filtration. Culture media were collected and immediately stored at $-20^{\circ} \mathrm{C}$ until chemical and enzymatic analyses. Then mycelia were rinsed with distilled water, freeze dried, weighed and stored at room temperature until chemical analysis.

\subsection{Enzymatic activity}

\subsubsection{Laccase in the culture medium}

Culture medium aliquots of $2.5 \mathrm{~mL}$ from flasks of batch \#4 and 6 were centrifugated at $9000 \mathrm{~g}$ for $20 \mathrm{~min}$ and $4{ }^{\circ} \mathrm{C}$ and the supernatant was used for the laccase assay. It was performed at $25^{\circ} \mathrm{C}$ in $2.9 \mathrm{~mL}$ of $100 \mathrm{mM}$ sodium acetate buffer, $\mathrm{pH} 4.5$, containing $2 \mathrm{mM}$ 2,6-dimethoxyphenol, plus $0.1 \mathrm{~mL}$ of the culture medium supernatant ( $3 \mathrm{~mL}$ final volume). The oxidation of 2,6-dimethoxyphenol was measured by spectrophotometry at $468 \mathrm{~nm}\left(\varepsilon_{468}=49600 \mathrm{M}^{-1} \mathrm{~cm}^{-1}\right.$; Perkin-Elmer Lambda 3B spectrophotometer). One unit of laccase activity was defined as the amount of enzyme required to oxidise $1 \mu \mathrm{mol}$ substrate $\min ^{-1}$ [20]. Results were analyzed by One-Way ANOVA.

\subsubsection{Effect of purified laccase on OTC degradation}

Direct OTC degradation by purified commercial laccase (from Trametes versicolor, $21.8 \mathrm{U} \mathrm{mg}^{-1}$, Sigma, Milan, Italy) was evaluated by incubating 50 or $100 \mu \mathrm{g} \mathrm{mL}^{-1}$ OTC with laccase $\left(0.1 \mathrm{U} \mathrm{mL}^{-1}\right.$ in $100 \mathrm{mM}$ sodium acetate buffer, $\mathrm{pH} 4.5$ ). Reaction was carried out under dark at $25^{\circ} \mathrm{C}$ in a final volume of $20 \mathrm{~mL}$ in amber flask, under continuous stirring. Control test was performed on OTC and buffer. Degradation was evaluated periodically up to $96 \mathrm{~h}$. OTC quantification was performed by direct injection of each sample in a LC-UV/DAD system (see Section 2.4.3).

\subsection{Chemical analysis}

\subsubsection{Extraction}

Mycelia were ground in a mortar, $100 \mathrm{mg} \mathrm{d}$. w. were poured into a $50 \mathrm{~mL}$ Falcon tube added with $100 \mu \mathrm{L}$ of a methanol solution of Doxycycline hyclate (DXC, Sigma, Milan, Italy) $100 \mu \mathrm{g} \mathrm{mL}^{-1}$ and extracted twice with $10 \mathrm{~mL}$ of $0.05 \mathrm{M}$ sodium acetate. After homogenization, sonication and mechanical agitation (15 min), the sample was centrifuged ( $5000 \mathrm{~g}, 5 \mathrm{~min}$, room temperature) and the pooled supernatants were cleaned-up on Metal Chelate Affinity Chromatography (MCAC) columns. Culture medium from each sample was filtered through a $0.45 \mu \mathrm{m}$ sterile membrane; $1 \mathrm{~mL}$

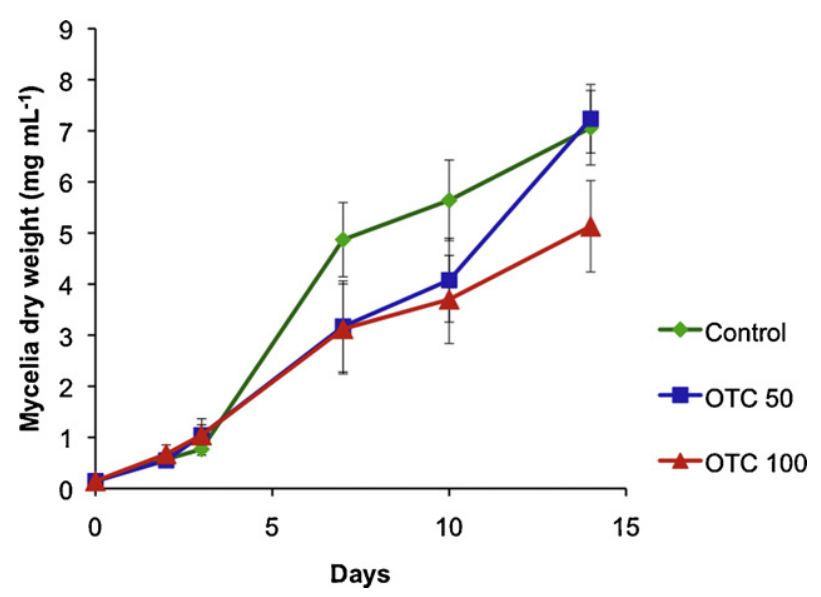

Fig. 1. Growth of $P$. ostreatus mycelium in control batch (green), $50 \mu \mathrm{g} \mathrm{mL}^{-1}$ OTC (blue) and $100 \mu \mathrm{g} \mathrm{mL}^{-1}$ OTC (red) exposed batches. (For interpretation of the references to color in this figure legend, the reader is referred to the web version of the article.)

of filtered sample was added with $100 \mu \mathrm{L}$ DXC methanol solution ( $\left.100 \mu \mathrm{g} \mathrm{mL}^{-1}\right)$ and cleaned-up on MCAC columns.

\subsubsection{Purification}

MCAC columns were prepared by filling $15 \mathrm{~mL}$ chromatography glass columns with $1.5 \mathrm{~mL}$ of Chelating Sepharose Fast Flow (GE Healthcare, Uppsala, Sweden). The sorbent was washed with $2 \mathrm{~mL}$ water:ethanol (80:20) solution and $6 \mathrm{~mL}$ water, conditioned with $3 \mathrm{~mL}$ of $0.01 \mathrm{M} \mathrm{CuSO}_{4}$ solution, then washed twice with $2 \mathrm{~mL}$ water. Columns were loaded with samples and then washed with $2 \mathrm{~mL}$ of water, then $2 \mathrm{~mL}$ methanol and $2 \mathrm{~mL}$ water. Elution was achieved with the application of $3 \mathrm{~mL}$ Mcllvaine buffer ( $\mathrm{pH} 4.5$ ). $20 \mu$ L eluate were injected into the HPLC-UV/DAD system.

In LC-MS analysis, $3 \mathrm{~mL}$ samples (in Mcllvaine buffer) were concentrated with SPE (Solid-Phase Extraction) polymeric columns (Oasis HLB, $60 \mathrm{mg} 3 \mathrm{~mL}$, Waters), preconditioned with $3 \mathrm{~mL}$ methanol and $3 \mathrm{~mL}$ water. After sample loading the column was washed with $3 \mathrm{~mL}$ of water, then vacuum dried. Elution was achieved with the application of $3 \mathrm{~mL}$ methanol, sample were dried under nitrogen flow and reconstituted in $100 \mu \mathrm{L}$ methanol. $20 \mu \mathrm{L}$ sample were injected onto the LC-ESI-MS system.

\subsubsection{HPLC-UV/DAD analysis}

An Agilent 1100 series HPLC system equipped with a RP XTerra MS $C_{8}$ column $(5 \mu \mathrm{m}, 2.1 \mathrm{~mm} \times 150 \mathrm{~mm})$ and photodiode array detector (set at $365 \mathrm{~nm}$ ) was used. Mobile phases were: A, 1\% acetic acid in water $(\mathrm{v} / \mathrm{v})$ and $\mathrm{B}$, acetonitrile HPLC grade. Flow rate: $0.3 \mathrm{~mL} \mathrm{~min}^{-1}$. Course gradient was: $0^{\prime}-2.5^{\prime} 2 \% \mathrm{~B}, 2.5^{\prime}-10.0^{\prime} 100 \%$ $\mathrm{B}, 10.0^{\prime}-12.0^{\prime} 100 \% \mathrm{~B}, 12.0^{\prime}-13.00^{\prime} 2 \% \mathrm{~B}, 13.0^{\prime}-15.00^{\prime} 2 \% \mathrm{~B}$. The estimated Limit of Detection (LOD) was $1 \mathrm{ppm}$.

\subsubsection{LC-ESI-MS analysis}

Liquid chromatography-electrospray ionization-mass spectrometry (LC-ESI-MS) analyses were performed with a Agilent 1100 series LC-MSD Trap mass spectrometer coupled to the HPLC system previously described, working at the same conditions. Analytes were detected using ESI in positive mode.

\section{Results}

\subsection{Fungal growth}

The growth of each experimental culture at different times is reported in Fig. 1 as mycelia dry weight. Both control and test 


\section{CONTROL (T3)}

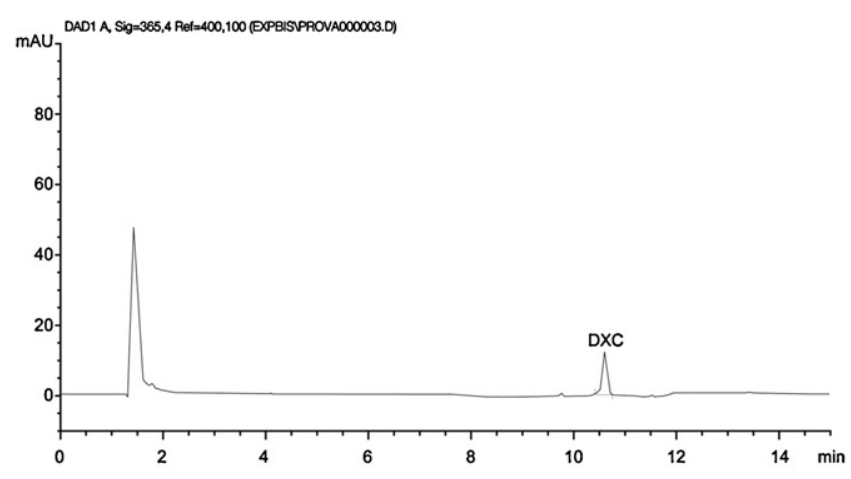

\section{OTC $50 \mu \mathrm{g} \mathrm{ml}^{-1}$}

OTC $100 \mu \mathrm{g} \mathrm{ml}^{-1}$
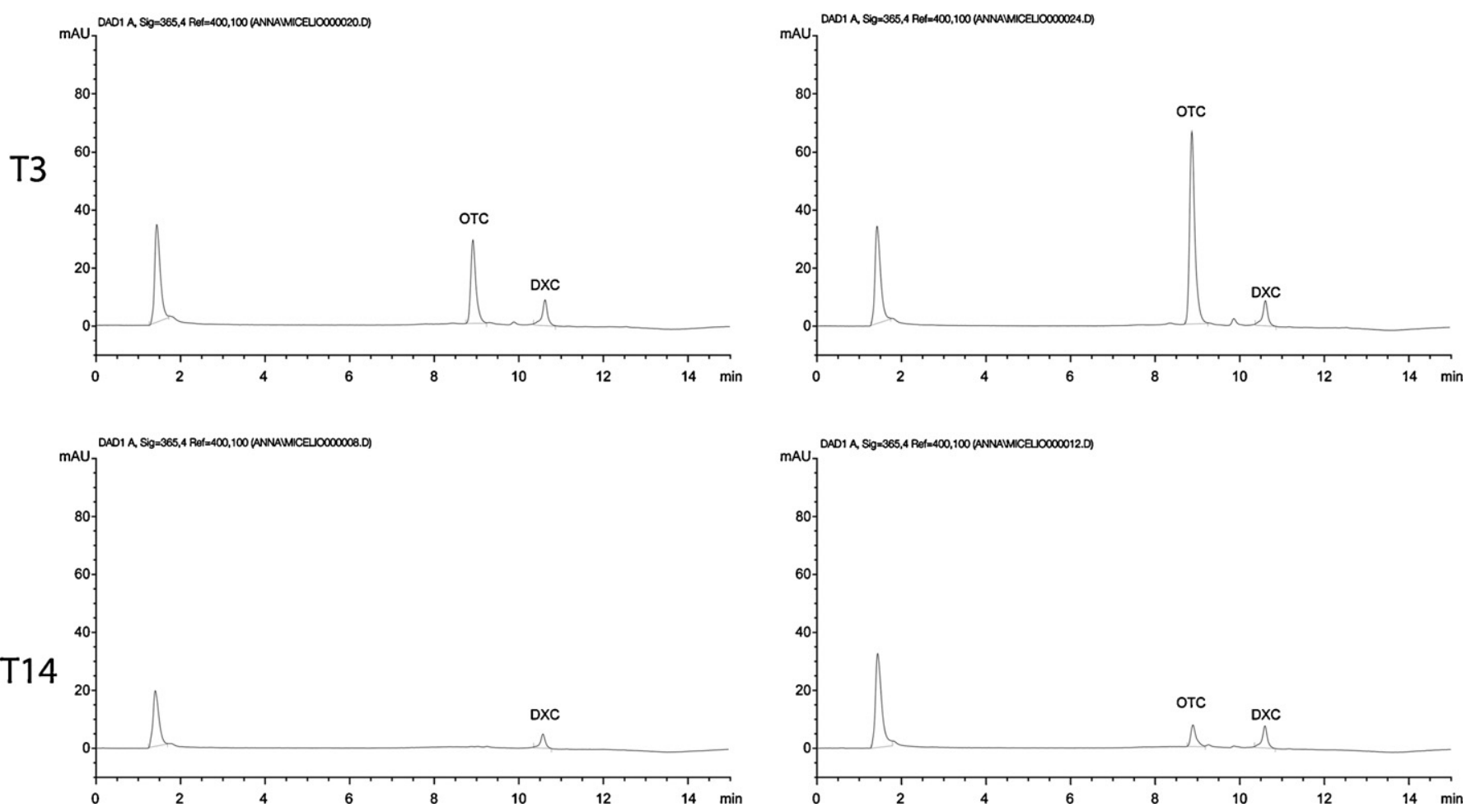

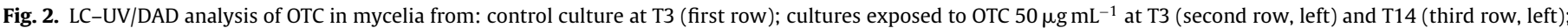
cultures exposed to OTC $100 \mu \mathrm{g} \mathrm{mL} \mathrm{m}^{-1}$ at T3 (second row, right) and T14 (third row, right). OTC= Oxytetracycline; DXC=Doxycycline (internal standard).

cultures showed active growth during the entire experimental period. After $14 \mathrm{~d}$ (T14) control cultures showed a 50-fold increase, from the initial $0.14 \mathrm{mg} \mathrm{mL}^{-1}$ of the inoculum to a final mean weight of $7.06 \mathrm{mg} \mathrm{mL}^{-1}$. The $50 \mu \mathrm{g} \mathrm{mL}^{-1}$ OTC culture reached the same final weight increase at T14, although weight is clearly lower than control until T10. Over the entire test the $100 \mu \mathrm{g} \mathrm{mL} \mathrm{L}^{-1}$ OTC culture showed lower fungal weight than the control. At T14 fungal growth reached a 36 -fold increase from the initial inoculum, to a final mean weight of $5.13 \mathrm{mg} \mathrm{mL}^{-1}$.

\subsection{OTC degradation}

OTC in the different batches and at different times was quantified by LC-UV/DAD analysis. The chemical analysis of the extract from control culture media and mycelia (batches \#1 and 4) excluded the presence of interfering peaks in the area of interest of the chromatograms. Analysis of culture media containing 50 or $100 \mu \mathrm{g} \mathrm{mL}^{-1}$ OTC without mycelium showed almost the same OTC concentrations from T0 to T14, demonstrating that OTC abiotic degradation did not occur in neither batch \#2 or 3 under these experimental conditions. On the contrary, the analysis of culture media and mycelia extracts from OTC exposed cultures (batches \#5 and 6) showed a dramatic reduction of OTC concentration in the course of the experiment (Fig. 2 and Fig. 3).

In particular, culture media of batch \#5 showed a dramatic OTC reduction in the first $7 \mathrm{~d}$ and a further reduction in the last $7 \mathrm{~d}$, up to an almost complete absence of OTC residues at T14 $\left(0.6 \mu \mathrm{g} \mathrm{mL}^{-1}\right)$. Contemporary, in batch \#5 mycelia, T2 and T3 extracts showed an increase of OTC content, followed by a progressive decrease up to the complete absence at T14 (Fig. 3). 


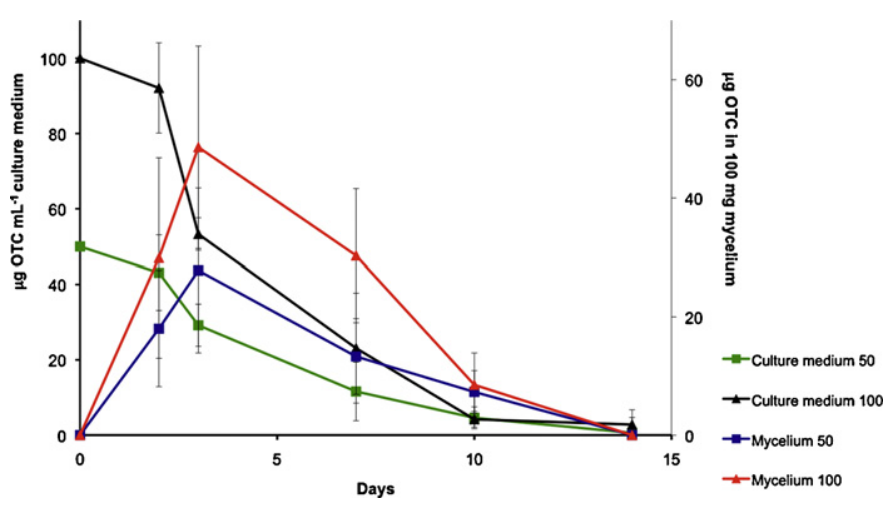

Fig. 3. OTC concentrations determinated by HPLC-UV/DAD in culture medium or mycelium at different times in 50 and $100 \mu \mathrm{g} \mathrm{mL}^{-1}$ OTC exposed batches.

A comparable trend of OTC degradation was found also in batch \#6. In the culture media, a sharp reduction of the initial concentration in the first $7 \mathrm{~d}$ and a further limited reduction in the last $7 \mathrm{~d}$ occurred; as a result a very low OTC concentration was found at T14 $\left(2.8 \mu \mathrm{g} \mathrm{mL}^{-1}\right)$. In mycelia extracts an initial increase of OTC content was found (T2-T3), followed by a progressive significant decrease that reduced the OTC residue at T14-7.6 $\mu \mathrm{g}$ per $100 \mathrm{mg}$ mycelium (d.w.; Fig. 3).

\subsection{Laccase activity in the culture medium}

The laccase activity during the degradation process was measured in the culture media of control (batch \#4) and OTC exposed culture (batches \#6; Fig. 4). P. ostreatus showed the production of extracellular laccase in the course of the trial, the maximum values being reached at T4-T5. No significant differences were obtained between control and OTC $100 \mu \mathrm{g} \mathrm{mL}^{-1}$, although a slightly higher activity was recorded from T3 to T5 in the presence of OTC.

\subsection{Effect of purified laccase on OTC degradation}

The degradation capability of the extracellular enzyme alone on OTC was tested by an in vitro test, coupled to OTC quantification. OTC solutions prepared according to the experimental conditions

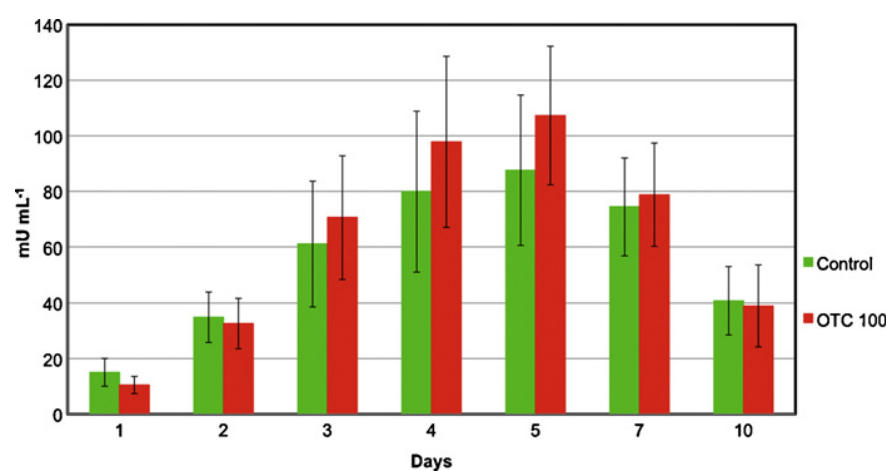

Fig. 4. Laccase activity measured in the culture medium of control and $100 \mu \mathrm{g} \mathrm{mL}^{-1}$ OTC treated batch.

\section{Table 1}

Percentage of OTC degradation due to purified commercial laccase. The results were produced by comparing the amount of OTC degraded in the batches with or without added laccase at each time.

\begin{tabular}{cll}
\hline Time (h) & OTC $50 \mu \mathrm{g} \mathrm{m}^{-1}(\%)$ & OTC $100 \mu \mathrm{g} \mathrm{mL}^{-1}(\%)$ \\
\hline 0 & 2.2 & 1.0 \\
1 & 1.8 & 0.5 \\
2 & 2.4 & 0.8 \\
3 & 2.3 & 1.3 \\
5 & 3.0 & 0.4 \\
24 & 2.4 & 0 \\
48 & 4.5 & 0.9 \\
72 & 6.6 & 0 \\
96 & 5.0 & 1.6 \\
\hline
\end{tabular}

reported in Section 2.3.2 were incubated with or without laccase. No significant differences in OTC degradation were measured in the two batches (Table 1 ).

\subsection{Products of OTC fungal degradation}

OTC degradation has been followed within the mycelial matrix using LC-ESI-MS full scan analysis of the extracts to detect the possible presence of degradation products. The results show three partially overlapping peaks (Fig. 5, coloured chromatogram). The first one (red) shows an OTC precursor ion $[\mathrm{M}+\mathrm{H}]^{+} \mathrm{m} / \mathrm{z} 461$, the second one (green) shows a precursor ion $[\mathrm{M}+\mathrm{H}]^{+} \mathrm{m} / \mathrm{z} 460$, that

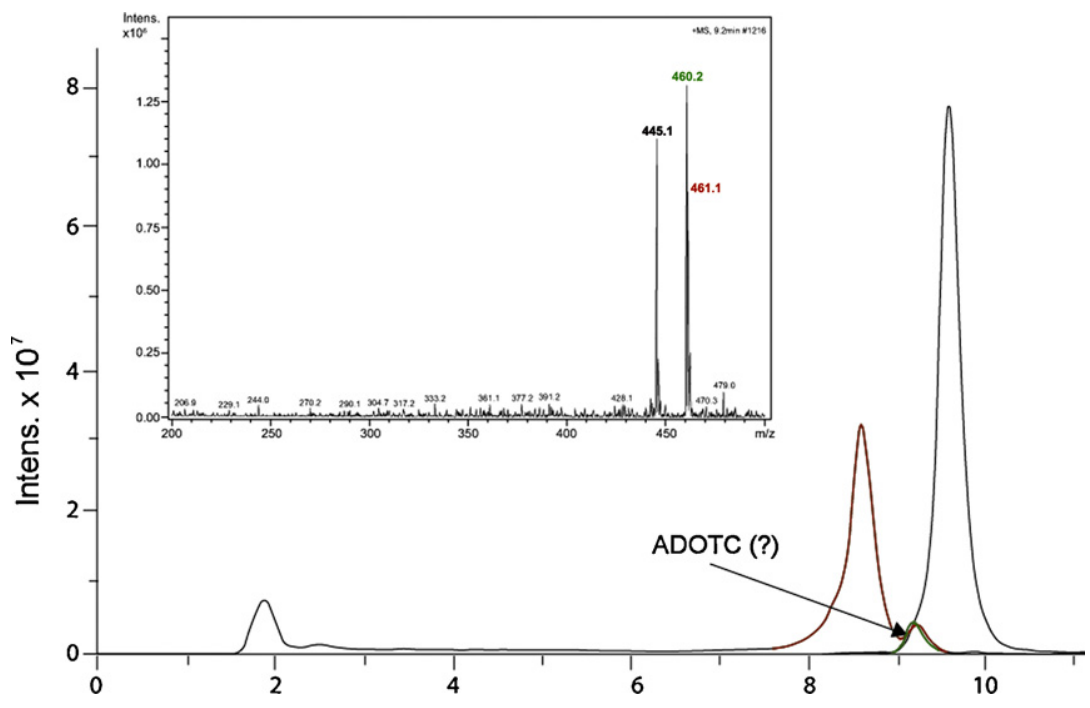

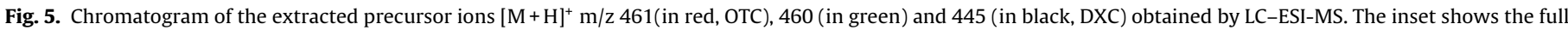

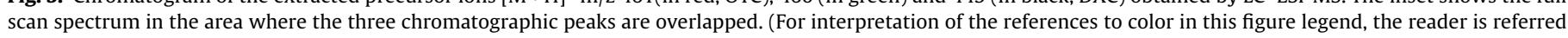
to the web version of the article.) 
a)

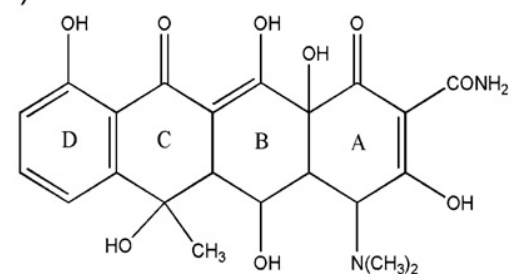

b)

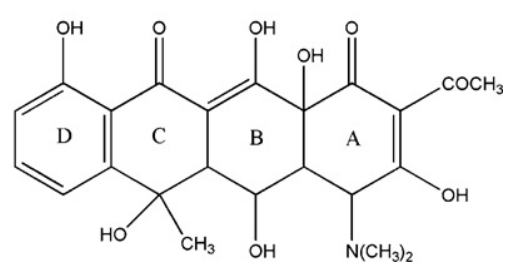

Fig. 6. Chemical structure of OTC (a) and ADOTC, 2-Acetyl-2-Decarboxamido-Oxytetracycline (b).

could be a degradation product of OTC, and the third one (black) a DXC precursor ion $[\mathrm{M}+\mathrm{H}]^{+} \mathrm{m} / \mathrm{z} 445$ (internal standard) (Fig. 5, inset).

\section{Discussion}

The proposed experimental model allows to demonstrate that (i) P. ostreatus is able to survive and grow in the presence of OTC, and (ii) the growing fungus actively removes (and degrades) the drug from the culture medium.

The growth dynamics of control and OTC exposed cultures shows that $P$. ostreatus mycelia survive and grow even in the presence of considerable amount of drug, although the growth rate is reduced at the higher drug concentration. In fact, at $50 \mu \mathrm{g} \mathrm{mL}^{-1}$, the rapid removal/degradation of the drug allows the mycelium to grow reaching the same final values of the control. At $100 \mu \mathrm{g} \mathrm{mL}^{-1}$, the mycelium starts a fast growth just after the removal/degradation of a considerable amount of drug.

While $\mathrm{pH}$ and UV can induce epimerization and isomerization reactions in the molecule [21], at our experimental conditions OTC does not undergo abiotic modifications. Drug removal (and degradation) from the culture medium is carried out by the growing fungus. In fact, after $7 \mathrm{~d}$ OTC in the culture media drops to $23 \%$ of the spiked drug, at both concentrations and the reduction still continues reaching very low amounts $(<3 \%)$ in the subsequent $7 \mathrm{~d}$ of experiment. While OTC is removed by the culture media, appreciable amounts of drug can be found within the mycelium (after $3 \mathrm{~d}$ exposure), demonstrating the OTC uptake by the fungus. Thus, the OTC reduction in the culture media has to be ascribed to the fungus, able to uptake the drug. The uptake is soon followed by an efficient degradation of the drug, being the amounts of OTC detected in the mycelia a spare fraction of the drug supplied.

To date these results on OTC cannot be compared with other experimental data, although $P$. ostreatus is known to absorb and degrade recalcitrant compounds [18,22]. In a study devoted to fluoranthene degradation, as a first step the compound was completely absorbed by the $P$. ostreatus mycelium (15d) and then degraded to $50 \%$ of the spiked amount in $40 \mathrm{~d}$ [23]. In a study on phenanthrene degradation using $P$. ostreatus, phase I and II metabolites were screened; the results indicated that the phase II enzymes, as Glutatione S-transferase, were not important for the elimination of hydroxylated phenanthrene [24].

As regard the involvement of laccase in OTC degradation under our experimental conditions, degradation test showed that laccase by itself cannot be considered as the prior responsible for fungal degradation of OTC, being unable to degrade OTC in the absence of mycelia. This result is in agreement with the experiments of Wen et al. [16,17], which demonstrated the degradation activity of a crude extract in which several enzymes and redox mediators were present.

The drug degradation process within the mycelial matrix has been shown in its early phases: LC-ESI-MS analyses allowed to follow the first degradation step of mycelia on the OTC molecule (Fig. 6a). In fact, in addition to the expected OTC precursor ion $\left([\mathrm{M}+\mathrm{H}]^{+} \mathrm{m} / \mathrm{z} 461\right)$, the mass spectrum revealed the presence of another precursor ion $\left([\mathrm{M}+\mathrm{H}]^{+} \mathrm{m} / \mathrm{z} 460\right)$; this additional precursor ion could be interpreted as 2-acetyl-2-decarboxamidooxytetracycline (ADOTC; $M W=459$; Fig. 6b). This compound is known to be a side product of OTC production by Streptomyces rimosus [25]. Its presence was analytically excluded in the standard solutions as impurity, but was revealed in the treated batches, only in the first days of the experiment. ADOTC has lower antibacterial potency on environmental relevant bacteria and higher lipophilicity than OTC [25]. These chemical characteristics determine a lower toxicity and environmental mobility, fulfilling the aim of our bioremediation approach.

\section{Conclusions}

This approach represents a first and successful step towards a feasibile and unexpensive bioremediation technique to avoid OTC spreading into environmental matrices.

$P$. ostreatus SMR684 represents a useful tool for OTC mycoremediation from contaminated matrices: the fungus is not only able to survive and grow in the presence of significant amount of drug, but also almost completely degrades the drug in few days. The drug is absorbed by the mycelia, where the OTC molecule undergoes a degradation step, as demonstrated by mass spectrometry analyses. In the treated mycelia no OTC-like residues are detected at the end of the experiment.

\section{Acknowledgments}

The Authors are grateful to Alessandro Pezzella, University of Naples "Federico II", for his insightful comments on the chemical aspects of this work. Work supported by RFO (Progetti di Ricerca Fondamentale Orientata) grant from Tor Vergata University to LM.

\section{References}

[1] P. Nielsen, N. Gyrd-Hansen, Bioavailability of oxytetracycline, tetracycline and chlortetracycline after oral administration to fed and fasted pigs, J. Vet. Pharmacol. Ther. 19 (1996) 305-311.

[2] S. Chiayvareesajja, A. Chandumpai, Y. Theapparat, D. Faroongsarng, The complete analysis of oxytetracycline pharmacokinetics in farmed pacific white shrimp (Litopenaeus vannamei), J. Vet. Pharmacol. Ther. 29 (2006) 409-414.

[3] G. Brambilla, M. Patrizii, S.P. De Filippis, G. Bonazzi, P. Mantovi, D. Barchi, L. Migliore, Oxytetracycline as environmental contaminant in arable lands, Anal. Chim. Acta 586 (2007) 326-329.

[4] F. Capolongo, A. Santi, L. Tomasi, P. Anfossi, M. Missagia, C. Montesissa, Residues of oxytetracycline and 4(-epimer in edible tissues from turkeys, J. AOAC Int. 85 (2002) 8-14.

[5] M. De Liguoro, V. Cibin, F. Capolongo, B. Halling-Sørensen, C. Montesissa, Use of oxytetracycline and tylosin in intensive calf farming: evaluation of transfer to manure and soil, Chemosphere 52 (2003) 203-212.

[6] L. Nepejchalovà, Z. Svobodovà, J. Kolařovà, K. Fragolvà, J. Valovà, D. Némethovà, Oxytetracycline assay in pond sediment, Acta Vet. Brno 77 (2008) 461-466.

[7] L. Migliore, F. Godeas, S.P. De Filippis, P. Mantovi, D. Barchi, C. Testa, N. Rubattu, G. Brambilla, Hormetic effect(s) of tetracyclines as environmental contaminant on Zea mays, Environ. Pollut. 158 (2010) 129-134.

[8] Q. Yang, J. Zhang, K. Zhu, H. Zhang, Influence of oxytetracycline on the structure and activity of microbial community in wheat rhizosphere soil, J. Environ. Sci. 21 (2009) 954-959. 
[9] B. Halling- Sørensen, S. Nors Nielsen, P.F. Lanzky, F. Ingerslev, H.C. Holten Lützhøfl, S.E. Jørgensen, Occurrence, fate and effects of pharmaceutical substances in the environment - a review, Chemosphere 36 (1998) 357-393.

[10] S. Kim, J.N. Jensen, D.S. Aga, A. Scott Weber, Tetracycline as a selector for bacteria in activated sludge, Chemosphere 66 (2007) 1643-1651.

[11] S. Thiele-Bruhn, I.C. Beck, Effects of sulfonamide and tetracycline antibiotics on soil microbial activity and microbial biomass, Chemosphere 59 (2005) 457-465.

[12] A.J. Williams, J. Deck, J.P. Freeman, M.P. Chiarelli, M.D. Adjej, T.M. Heinze, J.B. Sutherland, Biotransformation of flumequine by the fungus Cunninghamella elegans, Chemosphere 67 (2007) 240-243.

[13] G. Palmieri, G. Cennamo, G. Sannia, Remazol Brilliant Blue R decolourisation by the fungus Pleurotus ostreatus and its oxidative enzymatic system, Enzyme Microb. Technol. 36 (2005) 17-24.

[14] A.K. Haritash, C.P. Kaushik, Biodegradation aspects of polycyclic aromatic hydrocarbons (PAHs): a review, J. Hazard. Mater. 169 (2009) 1-15.

[15] H. Patel, A. Gupte, S. Gupte, Biodegradation of fluoranthene by basidiomycetes fungal isolate Pleurotus ostreatus HP-1, Appl. Biochem. Biotechnol. 157 (2009) 367-376.

[16] X. Wen, Y. Jia, J. Li, Degradation of tetracycline and oxytetracycline by crude lignin peroxidase prepared from Phanerochaete chrysosporium - a white rot fungus, Chemosphere 75 (2009) 1003-1007.

[17] X. Wen, Y. Jia, J. Li, Enzymatic degradation of tetracycline and oxytetracycline by crude manganese peroxidase prepared from Phanerochaete chrysosporium, J. Hazard. Mater. 177 (2010) 924-928.
[18] E. Galli, E. Brancaleoni, F. Di Mario, E. Donati, M. Frattoni, C.M. Polcaro, P. Rapanà, Mycelium growth and degradation of creosote-treated wood by basydiomycetes, Chemosphere 72 (2008) 1069-1072.

[19] P. Baldrian, Fungal laccases - occurrence and properties, FEMS Microbiol. Rev. 30 (2006) 215-242.

[20] C. Johannes, A. Majcherczyk, Laccase activity tests and laccase inhibitors, J. Biotechnol. 78 (2000) 193-199.

[21] P. Eichhorn, D.S. Aga, Identification of a photooxygenation product of Chlortetracycline in hog lagoons using LC/ESI-ion trap-ms and LC/ESI-timeof-flight-ms, Anal. Chem. 76 (2004) 6002-6011.

[22] C.M. Polcaro, E. Brancaleoni, E. Donati, M. Frattoni, E. Galli, L. Migliore, P. Rapanà, Fungal bioremediation of creosote-treated wood: a laboratory scale study on creosote components degradation by Pleurotus ostreatus mycelium, Bull. Environ. Contam. Toxicol. 81 (2008) 180-184

[23] S. Bobone, E. Donati, E. Galli, C.M. Polcaro, P. Rapanà, Biodegradation of fluoranthene by Pleurotus ostreatus, in: N. Kalogerakis, F. Fava, S.A. Banwart (Eds.) Proceedings of 4th European Bioremediation Conference, 3-6 September 2008, Chania, Crete, Greece, 2008, ID061.

[24] L. Bezalel, Y. Hadar, C.E. Cerniglia, Enzymatic mechanisms involved in phenanthrene degradation by the white rot fungus Pleurotus ostreatus, Appl. Environ. Microbiol. 63 (1997) 2495-2501.

[25] A.K. Lykkeberg, G. Sengeløv, C. Cornett, J. Tjørnelund, S.H. Hansen, B. Halling-Sørensen, Isolation structural elucidation and in vitro activity of 2-acetyl-2-decarboxamido oxytetracycline against environmental relevan bacteria, including tetracycline-resistant bacteria, J. Pharm. Biomed. Anal. 34 (2004) 559-567. 